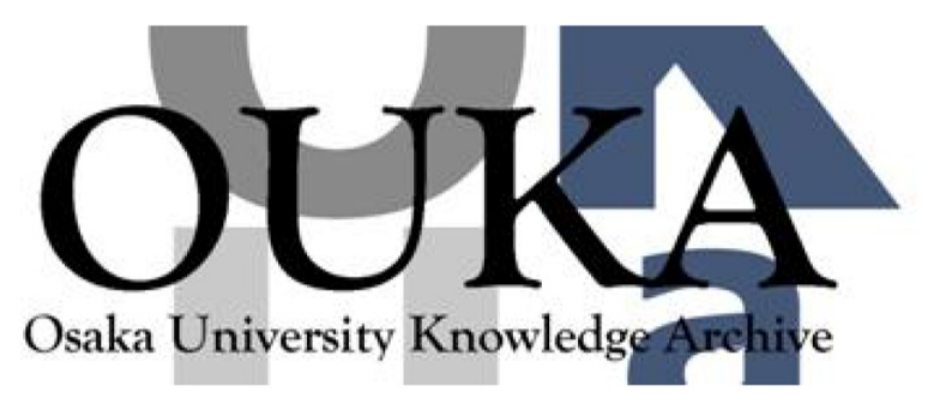

\begin{tabular}{|c|l|}
\hline Title & $\begin{array}{l}\text { Guiding and confining fast electrons by } \\
\text { transient electric and magnetic fields with a } \\
\text { plasma inverse cone }\end{array}$ \\
\hline Author(s) & Lei, A.L.; Cao, L. H. ; Yang, X.Q. et al. \\
\hline Citation & Physics of Plasmas. 16(2) p.020702 \\
\hline Issue Date & $2009-02$ \\
\hline oaire:version & VoR \\
\hline URL & https://hdl. handle.net/11094/3076 \\
\hline rights & \\
\hline Note & \\
\hline
\end{tabular}

Osaka University Knowledge Archive : OUKA

https://ir. Library. osaka-u. ac. jp/

Osaka University 


\title{
Guiding and confining fast electrons by transient electric and magnetic fields with a plasma inverse cone
}

\author{
A. L. Lei, ${ }^{1,2, a)}$ L. H. Cao, ${ }^{3}$ X. Q. Yang ${ }^{1}$ K. A. Tanaka, ${ }^{2}$ R. Kodama, ${ }^{2}$ X. T. He, ${ }^{3}$ K. Mima, ${ }^{4}$ \\ T. Nakamura, ${ }^{4}$ T. Norimatsu, ${ }^{4}$ W. Yu, and W. Y. Zhang ${ }^{3}$ \\ ${ }^{1}$ Shanghai Institute of Optics and Fine Mechanics, Chinese Academy of Sciences, Shanghai 201800, China \\ ${ }^{2}$ Institute of Laser Engineering and Graduate School of Engineering, Osaka University, \\ Osaka 565-0871, Japan \\ ${ }^{3}$ Institute of Applied Physics and Computational Mathematics, Beijing 100088, China \\ ${ }^{4}$ Institute of Laser Engineering, Osaka University, Osaka 565-0871, Japan
}

(Received 6 October 2008; accepted 7 January 2009; published online 5 February 2009)

\begin{abstract}
The fast electron propagation in an inverse cone target is investigated computationally and experimentally. Two-dimensional particle-in-cell simulation shows that fast electrons with substantial numbers are generated at the outer tip of an inverse cone target irradiated by a short intense laser pulse. These electrons are guided and confined to propagate along the inverse cone wall, forming a large surface current. The propagation induces strong transient electric and magnetic fields which guide and confine the surface electron current. The experiment qualitatively verifies the guiding and confinement of the strong electron current in the wall surface. The large surface current and induced strong fields are of importance for fast ignition related researches. (C) 2009 American Institute of Physics. [DOI: 10.1063/1.3075928]
\end{abstract}

Fast electrons with substantial numbers generated in the intense laser interactions ${ }^{1}$ have many potential applications, such as fast ignition (FI) of inertial confinement fusion. ${ }^{2}$ In the FI scheme, the fast electrons propagate into a precompressed core plasma and deposit their energy there for rapid heating to trigger a nuclear fusion burn wave. ${ }^{3,4}$ This scheme relies on the efficient propagation of fast electrons in the high density plasmas. The propagation process involves three dimensional complex relativistic nonlinear interactions of large current fast electrons with dense plasmas, making the detailed theoretical modeling of the fast electron transport difficult. ${ }^{5-8}$ Solid understanding on the propagation of large fast electron currents in simple targets is essential for the design of an effective FI target. ${ }^{5}$ Recently, the invention of the plasma fiber enables one dimensional guiding and collimation of the fast electrons, ${ }^{9}$ which may simplify the modeling and thus give detailed information on the electron transport in high density plasmas. ${ }^{10}$ The transition of fast electron transport from one dimension to two dimension (i.e., from a line to a surface) may further help understand the transport process and benchmark the numerical codes for scaling energy requirement of future FI laser facilities. ${ }^{11}$ It is therefore experimentally desired to guide and confine fast electrons to propagate along a surface for detailed numerical modeling. Surface current of fast electrons, in addition, may play an important role in the specific cone-in-shell target designed for FI. ${ }^{3}$ Sentoku et al. showed in a particle-in-cell (PIC) simulation that the fast electrons generated at the inner wall of a cone are guided to propagate along the wall surface toward the cone end, ${ }^{12}$ which might enhance the heating of the core plasma. This surface current was observed with a planar target in both simulation ${ }^{13}$ and experiments. ${ }^{14,15}$ However, with a cone target configuration, the guided electrons

${ }^{a)}$ Electronic mail: lal@siom.ac.cn. propagating along the cone wall surface will mix the electrons generated at the cone end by the laser light, and hence one cannot experimentally quantify the numbers of guided fast electrons by the cone wall. The guided fast electrons will also mix the electrons generated ahead by the laser light with a planar target.

We here report a novel surface current of fast electrons propagating in high density plasmas shaped in an inverse cone. The outer tip of the inverse cone faces the incident laser and the inner tip faces the void beneath it. The intense laser irradiates the outer tip, generating fast electrons. The fast electrons are then guided and confined to propagate along the inverse cone wall to form a surface current by the induced transient electric and magnetic fields associated with the current itself. Once departing from the source at the outer tip, this surface current of fast electrons is "clean," neither experiencing the interacting laser light nor mixing fast electrons ahead, unlike those in cone or planar targets. This surface current in the inverse cone is therefore useful for two dimensional modeling of the large current fast electron transport in high density plasmas, and may also explicitly give the capability of the guiding of fast electrons by the cone wall in a cone-in-shell target. ${ }^{3}$

The intense laser pulse is injected from the left axially onto the outer tip of an inverse cone plasma, producing fast electrons with substantial numbers and inducing strong transient electric and magnetic fields associated with the fast electron current, as shown in Figs. 1(a)-1(d). Figures 1(a)-1(d) show the distributions of the electric field $E_{y}$ and magnetic field $B_{z}$ in the inverse cone plasma at two different times, as generated by a two dimensional PIC simulation, ${ }^{16}$ showing the fast electron propagation being accompanied by these fields. Figure 1(e) shows the profiles of $E_{y}$ and $B_{z}$ at $x=15 \mu \mathrm{m}$ and $t=29.84 \tau$, where $\tau \approx 3.5$ fs is the laser period. The $p$-polarized $1.06 \mu \mathrm{m}$ laser pulse has a spatial pro- 

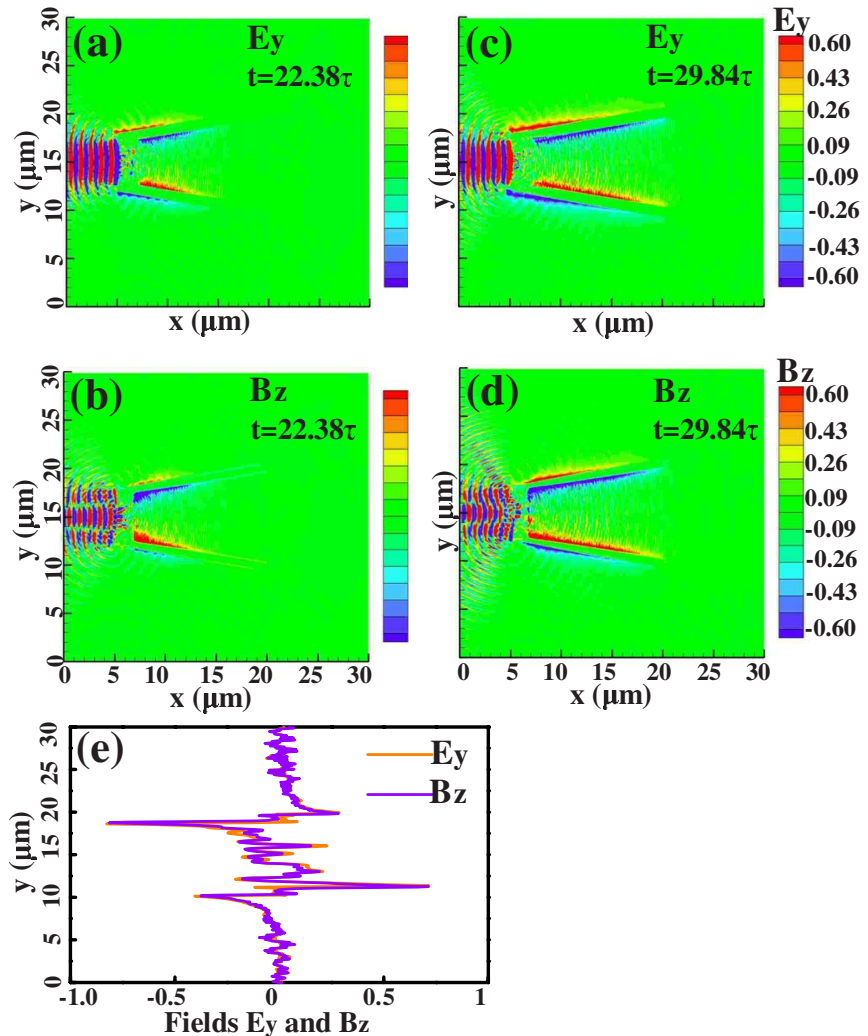

FIG. 1. (Color online) Two-dimensional PIC simulation demonstrating the guiding and confinement of the high density fast electrons with an intense laser irradiated plasma inverse cone. The fast electrons propagate along the inverse cone wall, inducing strong electric and magnetic fields. [(a)-(d)] are the distributions of the electric and magnetic fields $\left(E_{y}\right.$ and $\left.B_{z}\right)$ snapped at $22.38 \tau$ and $29.84 \tau$, respectively. (e) shows the strength profiles of $E_{y}$ and $B_{z}$ at $x=15 \mu \mathrm{m}$ at time $29.84 \tau$. $E_{y}$ and $B_{z}$ are in units of $3 \times 10^{10} \mathrm{~V} / \mathrm{cm}$ and $10^{8} \mathrm{G}$, respectively.

file $I_{0} \exp \left(-r^{2} / R^{2}\right)$, where $I_{0}=5 \times 10^{19} \mathrm{~W} \mathrm{~cm}^{-2}$ and $R$ $=1 \mu \mathrm{m}$. It has a temporal Gaussian leading edge of duration $4 \tau$, followed by a constant intensity $\left(I=I_{0}\right)$ plateau of duration $50 \tau$. The full open angle of the inverse cone is $20^{\circ}$. The diameter of the outer tip is $6 \mu \mathrm{m}$, the thicknesses of the tip and the wall are 2 and $1 \mu \mathrm{m}$, respectively. The initial plasma density is $10 n_{c}$, where $n_{c}$ is the critical density. The fast electrons are generated at the outer tip and they propagate forward into the inverse cone plasma as the background electron return current can compensate the charge neutrality. The propagation of the fast electrons induces both strong radial electric field of the order of $10^{10} \mathrm{~V} / \mathrm{cm}$ (combination of $E_{y}$ and $E_{x}$, and $E_{x}$ is not shown) and azimuthal magnetic field $B_{z}$ of the order of $10 \mathrm{MG}$ associated with the fast electron current. These fields, in turn, act to guide and confine the fast electrons to propagate along the inner and outer surfaces of the inverse cone wall, forming a strong surface current, as have been discussed by Kodama et al. ${ }^{9}$

Figure 2 shows the electron kinetic energy density $(\gamma$ $-1) n_{e} / n_{c}$ distributions snapped at time 59.67 $\tau$. Where $\gamma$ $=1 / \sqrt{1-v_{e}^{2} / c^{2}}$ and $n_{e}$ is the electron density. It is seen that the electron kinetic energy flux is along the inverse cone wall, showing surface guiding and confinement of the fast electrons, consistent with the electric and magnetic field contours shown in Figs. 1(a)-1(d).

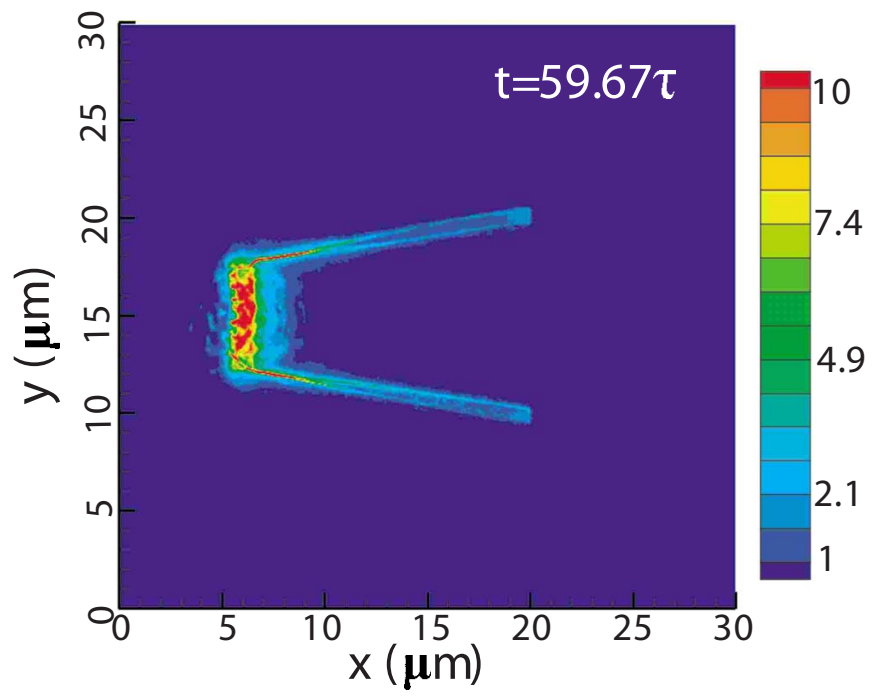

FIG. 2. (Color online) Electron energy density distributions snapped at time $59.67 \tau$. The color code is in unit of $0.511 \times 10^{21} \mathrm{MeV} / \mathrm{cm}^{3}$.

We now experimentally examine the guiding and confinement of the fast electrons with substantial numbers with an $\mathrm{Au}$ inverse cone target. The target and experimental setup are shown in Fig. 3(a). The inverse cone target had a $20^{\circ}$ full open angle. The outer and inner tip sizes were 100 and $80 \mu \mathrm{m}$ in diameter, the thicknessess of the tip and the wall were both $7 \mu \mathrm{m}$, and the length from the tip to the entrance was $600 \mu \mathrm{m}$, respectively. The experiment was performed on the Gekko XII PW (GXII PW) laser ${ }^{17}$ at the Institute of Laser Engineering, Osaka University. The angular distribution of fast electrons emitted forward was measured with a detector stack placed $30 \mathrm{~mm}$ behind the target. The stack consisted of several $12 \mu \mathrm{m}$ thick Al foils, $110 \mu \mathrm{m} \mathrm{CR39s,} \mathrm{a}$ $5 \mathrm{~mm}$ plastic plate, and two imaging plates (IPs) (Fuji BASSR2025) as the electron detector. ${ }^{18}$ The IPs were wrapped by the $\mathrm{Al}$ foils so as to prevent the optical light striking the IPs. The CR39s and the plastic plate may serve as the energy selector for the fast electrons. Electrons with energy higher than $1.8 \mathrm{MeV}$ were recorded on the second IP. In the experiment campaign, the $p$-polarized GXII PW laser $(\lambda$ $=1.053 \mu \mathrm{m}, \tau=0.6 \mathrm{ps}, E=90 \mathrm{~J}$ ) had a large focus size of 70-100 $\mu \mathrm{m}$ [full width at half maximum (FWHM)]. The GXII PW laser was focused on the outer tip of the inverse cone, at $26^{\circ}$ to the tip normal, with the most intense laser focal spot arranged to deviate from the outer tip of the inverse cone such that only the periphery of the focused laser spot irradiated one edge of the inverse cone outer tip. The main laser pulse thus directly hit the $12 \mu \mathrm{m}$ thick Al foil. This laser focus arrangement aimed at avoiding the laser irradiation on the outer wall of the inverse cone to generate fast electrons there, which may mix the electrons originating from the inverse cone outer tip, thus giving an explicit evidence of the guiding and confinement of the fast electrons coming from the inverse cone outer tip by the inverse cone wall, as shown in Figs. 1(a)-1(d).

Figure 3(b) shows the measured raw image of fast electrons with energy higher than $1.8 \mathrm{MeV}$. The image shows two circular shapes which overlap with each other. The sig- 

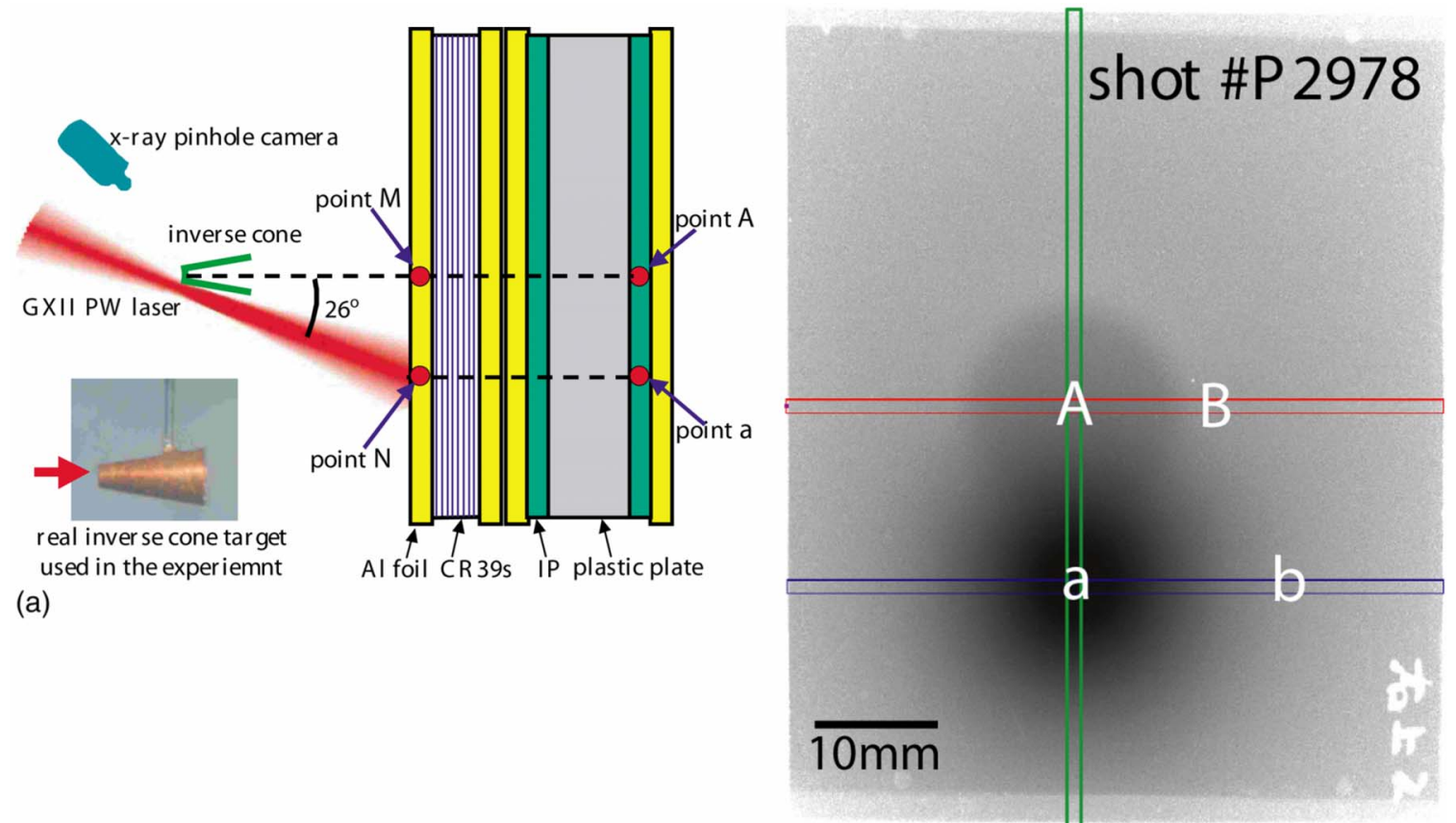

(b)

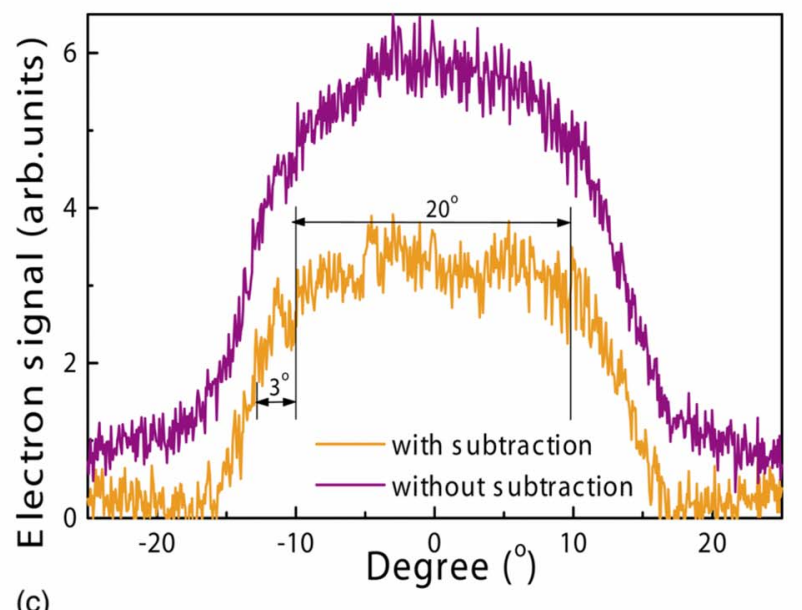

(c)

FIG. 3. (Color online) Experimental demonstration of the guiding and confinement of fast electrons with a plasma inverse cone. (a) is the experimental setup. The points $A$ and $a$ on the second IP in (a) nearly correspond to points $A$ and $a$ in (b), respectively. (b) shows the raw emission image of fast electrons with energy higher than $1.8 \mathrm{MeV}$. (c) are the emission intensity profiles of fast electrons extracted from the lane $A B$ in (b). The upper blue curve shows the raw emission intensity and the lower orange curve is the electron signal purely from the inverse cone target via subtracting the electron signal from the Al foil. The degree $0^{\circ}$ corresponds to point $A$ in (a), which is on the inverse cone axis.

nal of the upper circular is weaker and centers around point $A$. Point $A$ is on the inverse cone axis, determined by the target and detector alignment, indicating that the inverse cone target contributes to the electron emission in the upper circular shape. The signal of the lower circular is stronger and peaks around point $a$, and gradually fades out radially. Point $a$ in Fig. 3(b) nearly corresponds to the point $a$ in Fig. 3(a) which is behind the point $N$ that is on the GXII PW laser axis, indicating the lower circular emission is due to the laser direct irradiation on the $12 \mu \mathrm{m} \mathrm{Al}$ foil. The distance between point $a$ and $A$ is about $14 \mathrm{~mm}$, giving the angle between point $M$ and $N$ in Fig. 3(a) relative to the inverse cone target being $25^{\circ}$, nearly equal to the laser incidence angle $26^{\circ}$, consistent to the target, detector, and laser alignment. The fast electron emission from the inverse cone target has a sharp boundary as shown in Fig. 3(c) below, contrary to the fast electron emission from the Al foil shown as the lower circular in Fig. 3(b) where the electron signal gradually fades out radially. There is also no such sharp boundary in the fast electron emission observed with a flat Au target, ${ }^{19}$ indicating that there is a clear difference in the fast electron emission pattern between a flat target and the inverse cone target. With the inverse cone target the fast electron emission is confined within a solid angle determined by the sharp boundary shown in Fig. 3(b).

We obtain the fast electron signal purely from the inverse cone target via subtracting the electron signal from the $12 \mu \mathrm{m} \mathrm{Al}$ foil. We define that $S_{B}$ and $S_{b}$ are the electron signal intensities at point $B$ and $b$, respectively. Points $B$ and $b$ move along the lane $A B$ and lane $a b$ in Fig. 3(b) with $a B$ 
$=a b$. We assume that the fast electron emission from the Al foil is radially symmetric and peaks at point $a$ in Fig. 3(b). $\Delta S=S_{B}-S_{b}$ hence represents the electron signal at point $B$ purely from the inverse cone target. A profile of $\Delta S$ along the direction from point $B$ to point $A$ in the lane $A B$ in Fig. 3(b) is shown in Fig. 3(c) as the lower orange curve. The profile shows a sharp boundary. The fast electron emission angle from the peak intensity to the half-value is about $3^{\circ}$, comparable to $2.5^{\circ}$ for a plasma fiber ${ }^{9}$ and much less than $35^{\circ}$ for a planar Au target. ${ }^{19}$ The electron emission from the inverse cone target is within the sharp boundary which has a solid angle of about $22^{\circ}$, nearly equal to the inverse cone target open angle of $20^{\circ}$.

We discuss how the observed fast electron emission pattern in vacuum is related to the surface guiding and confinement of fast electrons with substantial numbers along the inverse cone wall inside the target. The fast electrons are generated at the outer tip of the inverse cone target and then propagate forward either to the inner tip or along the inverse cone wall surface. Electrons escaped from both the wall surface and inner tip can contribute to the observed emission on the IPs. First, electrons escaped from the wall surface are guided and therefore have a narrow beam divergence. This results in a ring pattern of fast electron emission. This emission ring has a sharp boundary which has a solid angle nearly equal to the inverse cone target open angle $20^{\circ}$. Second, the PIC simulation shows that strong transient electric and magnetic fields are induced on the inner wall surface by the substantial numbers of fast electrons propagating along the inverse cone wall surface, as indicated in Figs. 1(a)-1(d). These strong fields surround the path of the fast electrons escaped from the inner tip. Escaped fast electrons with initial divergence angle larger than $10^{\circ}\left(=20^{\circ} / 2\right)$ from the inner tip move toward the inner wall surface and experience the strong fields and thus are collimated and confined within the hollow inverse cone. This is the reason why the pattern of the fast electron emission from both the wall surface and inner tip has a sharp boundary which has a specific solid angle (i.e., about $22^{\circ}$ ) nearly equal to the inverse cone target open angle of $20^{\circ}$. Note the $1.8 \mathrm{MeV}$ electrons with divergence angle larger than $10^{\circ}$ from the inner tip cannot be confined or blocked by the $7 \mu \mathrm{m}$ thick Au inverse cone wall via classic stopping. With a planar foil target, we never observed such a sharp boundary of fast electron emission pattern, but usually a spatially Gaussian distribution with much larger electron beam divergence angle of $40^{\circ}-70^{\circ}$ (FWHM) ${ }^{19}$ The observed fast electron emission image qualitatively verifies the guiding and confinement of fast electrons with substantial numbers along the wall surface and indicates that the fast electrons escaped from the inner tip are collimated and confined by the induced fields.

In summary, we have observed the surface guiding and confinement of fast electrons with substantial numbers with a plasma inverse cone, which is due to the induced transient electric and magnetic fields. This surface electron current and induced fields could be experimentally diagnosed via fast electron collision or heating effect with various diagnostics including optical, extreme ultraviolet, $K \alpha$ x-ray, and en- ergetic particle imagings, ${ }^{9,10,20}$ for detailed modeling of the electron transport in high density plasmas, which is essential for FI. The surface current existing in the inverse cone wall could also give the capability of the guiding of fast electrons by the cone wall in a cone-in-shell target. Guiding and confining fast electrons with a plasma inverse cone might have several important applications in high energy density physics. One example is to generate collimated or even focused energetic ions, which are emitted from the inner tip into the hollow inverse cone. The inverse cone target is a combination of a foil target and a hollow cylinder target. The collimation of energetic ions with a plasma inverse cone is similar to that with a plasma hollow cylinder, ${ }^{21}$ but with a simpler configuration.

We are grateful for the helpful discussions with M. H. Key, P. Norreys, Y. Sentoku, and R. B. Stephens. We acknowledge all the technical support of the engineering staff at ILE for the laser operation, target fabrication, and data acquisition. This work was supported by the JSPS JapanChina Core University Program, the Core to Core Program on International Collaboration for High Energy Density Science, the Japan-Korea-China Cooperative Project on High Energy Density Sciences for Laser Fusion Energy, the Natural Science Foundation of China under Grant Nos. 10675024, 10775165, 10835003, and 10875158, the Science and Technology Commission of Shanghai Municipality under Grant No. 08PJ14102, and the National Basic Research Program of China under Grant Nos. 2007CB815101 and 2006 CB806004.

${ }^{1}$ M. D. Perry and G. Mourou, Science 264, 917 (1994).

${ }^{2}$ M. Tabak, J. Hammer, M. E. Glinsky et al., Phys. Plasmas 1, 1626 (1994).

${ }^{3}$ R. Kodama, P. A. Norreys, K. Mima et al., Nature (London) 412, 798 (2001).

${ }^{4}$ Y. Kitagawa, Y. Sentoku, S. Akamatsu et al., Phys. Rev. E 71, 016403 (2005).

${ }^{5}$ R. R. Freeman, D. Batani, S. Baton et al., Fusion Sci. Technol. 49, 297 (2006).

${ }^{6}$ M. H. Key, Phys. Plasmas 14, 055502 (2007).

${ }^{7}$ R. B. Campbell, R. Kodama, T. A. Mehlhorn et al., Phys. Rev. Lett. 94, 055001 (2005).

${ }^{8}$ R. J. Mason, Phys. Rev. Lett. 96, 035001 (2006).

${ }^{9}$ R. Kodama, Y. Sentoku, Z. L. Chen et al., Nature (London) 432, 1005 (2004).

${ }^{10}$ J. S. Green, K. L. Lancaster, K. U. Akli et al., Nat. Phys. 3, 853 (2007); J. J. Honrubia, ibid. 3, 835 (2007).

${ }^{11}$ M. Dunne, Nat. Phys. 2, 2 (2006).

${ }^{12}$ Y. Sentoku, K. Mima, H. Ruhl et al., Phys. Plasmas 11, 3083 (2004).

${ }^{13}$ T. Nakamura, S. Kato, H. Nagatomo, and K. Mima, Phys. Rev. Lett. 93, 265002 (2004).

${ }^{14}$ H. Habara, K. Adumi, T. Yabuuchi et al., Phys. Rev. Lett. 97, 095004 (2006).

${ }^{15}$ Y. T. Li, X. H. Yuan, M. H. Xu et al., Phys. Rev. Lett. 96, 165003 (2006).

${ }^{16}$ H. Xu, W. W. Chang, Y. Yin, and H. B. Zhuo, Chin. J. Comput. Phys. 20, 326 (2003).

${ }^{17}$ Y. Kitagawa, H. Fujita, R. Kodama et al., IEEE J. Quantum Electron. 40, 281 (2004).

${ }^{18}$ K. A. Tanaka, T. Yaabuchi, T. Sato et al., Rev. Sci. Instrum. 76, 013507 (2005).

${ }^{19}$ A. L. Lei, K. A. Tanaka, R. Kodama et al., Phys. Rev. Lett. 96, 255006 (2006).

${ }^{20}$ R. A. Snavely, B. Zhang, K. Akli et al., Phys. Plasmas 14, 092703 (2007).

${ }^{21}$ T. Toncian, M. Borghesi, J. Fuchs et al., Science 312, 410 (2006). 